\title{
Screening of Molecular Interactions Using Reporter Hammerhead Ribozymes
}

\author{
Jörg S. Hartig and Michael Famulok
}

\begin{abstract}
The characterization of molecular interactions is a central task in modern life sciences. Applications such as drug screening in pharmaceutics or the elucidation of biomolecular interactions in molecular biology rely on efficient methods to search for interacting partners. Here, we describe a novel technique that utilizes hammerhead ribozymes to signal molecular interactions. The ribozyme is modified by a domain that specifically binds to a target molecule such as a protein. Upon binding of the target, the catalytic activity of the ribozyme is changed, allowing for detection of the presence as well as the occurrence of interactions of the targeted ligand. The assay can be performed in high-throughput format by employing double-labeled ribozyme substrates, hence being well suited for drug-screening applications. The detection proceeds rapidly and in real-time. Moreover, the technique neither requires labeling of the target molecule nor the potential interaction partners or analytes since an indirect readout is facilitated by switching the catalytic activity of a reporter ribozyme. The assay can be utilized to sense a broad variety of biomolecular interactions, and is very sensitive due to signal amplification by the ribozyme reaction.
\end{abstract}

Key Words: Hammerhead ribozyme; aptamer; RNA reporter; drug-screening; proteininteractions; FRET-assay; signal amplification.

\section{Introduction}

Catalytic RNAs, also known as ribozymes, have been employed in the engineering of biosensors for the detection of target molecules (1). In these studies, phosphodiester-cleaving ribozymes are modified by aptamers. By attaching small molecule-binding aptamers to ribozymes, biosensors were generated that signal the presence of the ligand by changing the catalytic activity of the ribozyme $(1,2)$. Further work on such fusions of aptamers and ribozymes (so-called aptazymes, or reporter-ribozymes) resulted in the development of 

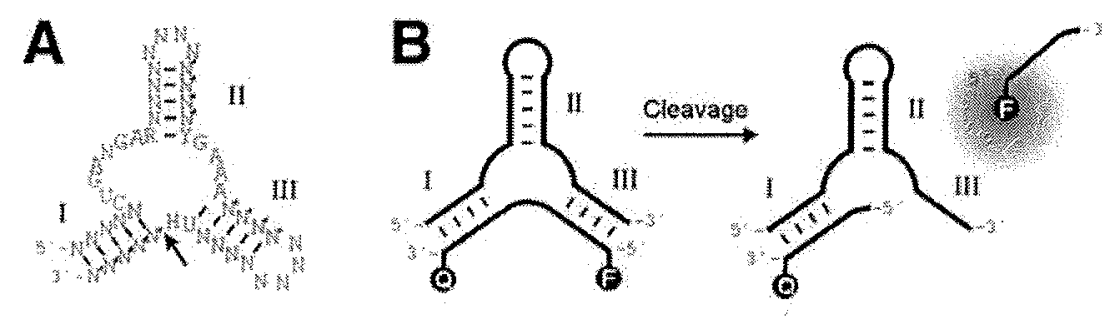

Fig. 1. The hammerhead ribozyme. (A) Secondary structure and conserved nucleotides. The ribozyme is shown in the intramolecular cleavage (in cis) format. The cleavage site is indicated by the arrow. $N$, any nucleotide; $N^{\prime}$, any nucleotide complementary to $N$; $H$, any nucleotide except $\mathrm{G} ; R$, purine nucleotide; $Y$, pyrimidine nucleotide complementary to $R$. (B) Schematic of a hammerhead ribozyme-cleaving intermolecular (in trans). A pair of FRET dyes is attached to the RNA substrate (bottom strand), enabling readout of cleavage activity via fluorescence detection. $F$, fluorescent dye; $Q$, fluorescence quencher.

biosensors capable of detecting interactions between proteins and other biomolecules. These assays are based on ribozymes that change their catalytic activity in the presence of the target protein. If the protein-bound ribozyme is incubated with an interaction partner of the protein, the ribozyme is displaced and again undergoes a change of catalytic activity (Fig. 2). Detection of the interaction is greatly facilitated by monitoring the ribozyme-catalyzed cleavage reaction of fluorescently labeled ribozyme substrates $(3,4)$. Upon cleavage of the substrate, a fluorescent signal is generated by separating a pair of fluorescence resonance energy transfer (FRET) dyes attached to the ribozyme substrate. Hence, the readout of fluorescence allows the detection of biomolecular interactions, rendering the method suitable for high-throughput analysis. Here, we introduce different strategies for the construction of protein-dependent hammerhead ribozymes (HHRs), capable of detecting protein interactions. The method can be used to identify interactions of the target protein with varying classes of molecules, such as nucleic acids, smaller molecules, as well as other proteins. For example, we have employed reporter ribozymes in screens detecting protein interactions with small molecules for drug-screening purposes (5).

The method described in this chapter exploits protein-regulated HHRs for the identification of biomolecular interactions. The HHR (see Fig. 1A) represents one of the best characterized catalytic RNAs. The HHR has relatively few requirements for catalytic activity: Its catalytic core, which is comprised of the conserved nucleotides shown in Fig. 1A, its three flanking variable helices, termed stems I, II, and III, and the presence of divalent metal ions such as $\mathrm{Mg}^{2+}$. A detailed discussion of HHR kinetics can be found in the literature $(6,7)$. Hammerhead cleavage can be performed to occur in trans (intermolecularly) by deleting the terminal loop of one of the stems (see Fig. 1B, generated by deletion 
of stem III loop in Fig. 1A). Carrying out the cleavage reaction in trans allows the convenient attachment of a pair of fluorescent reporter dyes to the RNA substrate. In this system, the fluorescence of one reporter dye in the intact RNA substrate is quenched by FRET to the second dye (see Fig. 1B, left). In addition to easy readout of the cleavage reaction, multiple turnover of the substrate results in signal amplification, which is helpful when investigating low-affinity interactions or samples with low concentrations of target molecules (8). A single interaction can trigger the catalytic activity of one ribozyme molecule, which then goes on to cleave multiple substrates, thereby amplifying the detection signal.

Ribozymes that change their catalytic activity in the presence of a target protein can be used to screen for interaction partners of the protein (see Fig. 2). To generate ribozymes that switch cleavage activity upon binding of the targeted molecule, protein-binding RNA sequences are connected to the ribozyme. So far, three distinct strategies for the construction of a protein-regulated aptazyme have been reported: direct selection, starting from partly randomized ribozyme pools $(1,9-12)$; selection of the connection site that links a preexisting aptamer and a ribozyme $(\mathbf{1 0 , 1 3 )}$; and rational design of aptazymes, lacking the selection step. Since the direct selection of an RNA enzyme responding to a protein has not yet been demonstrated and selection procedures for generation of aptazymes are still relatively complex and time-consuming, we will focus on the rational design of protein-dependent ribozymes for reporting molecular interactions.

\subsection{Rational Design of Reporter Hammerhead Ribozymes}

The technique of constructing an aptazyme by means of rational design is made possible by the often predictable and easily programmable structural features of nucleic acids, based on simple rules, such as Watson-Crick base pairing. Aptazymes have been constructed by simply fusing an existing aptamer to a ribozyme. For example, aptamers can be linked to a ribozyme sequence in a way that the aptamer hybridizes to the substrate recognition site of the ribozyme (see Fig. 3A). In order to facilitate the intramolecular interaction of the ribozyme's substrate-binding site with the aptamer sequence, the sequences of the substrate-binding site of the ribozyme, and also the substrate itself, can be altered. The HHR requires a substrate with the conserved sequence UH, where $\mathrm{H}$ is any nucleobase but $\mathrm{G}$ (see Fig. 1A). In addition, the cleavage site must be flanked by sequences that allow hybridization to the ribozyme so that the helical stem structures I and III are formed.

\subsubsection{Detecting Interactions of HIV-1 Rev}

For example, an aptamer sequence for the human-immunodeficiency virus type 1 (HIV-1) Rev protein was attached to the $5^{\prime}$-end of a HHR (5). In the absence of Rev protein, the aptamer sequence hybridizes to the $5^{\prime}$-end of the ribozyme, 
A
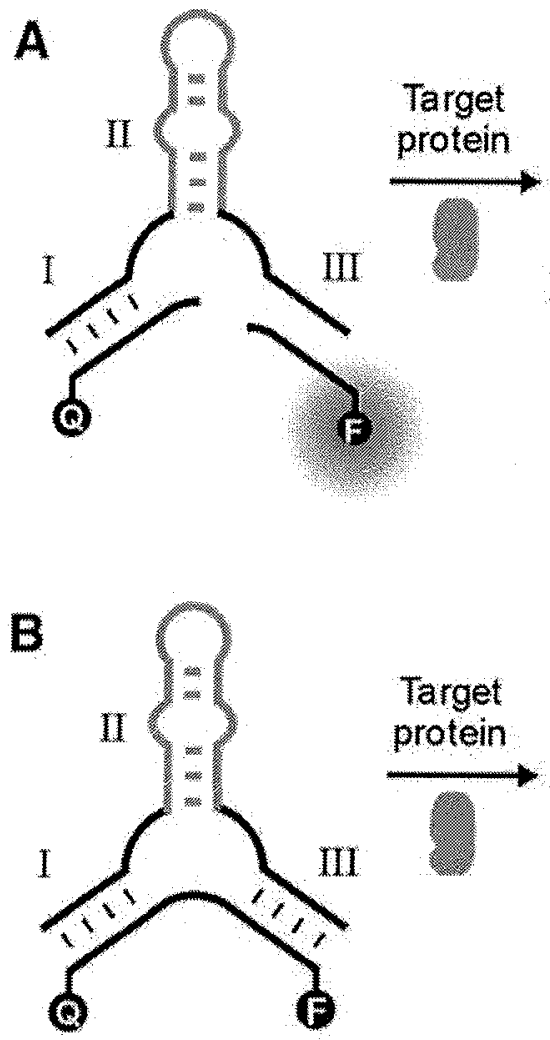
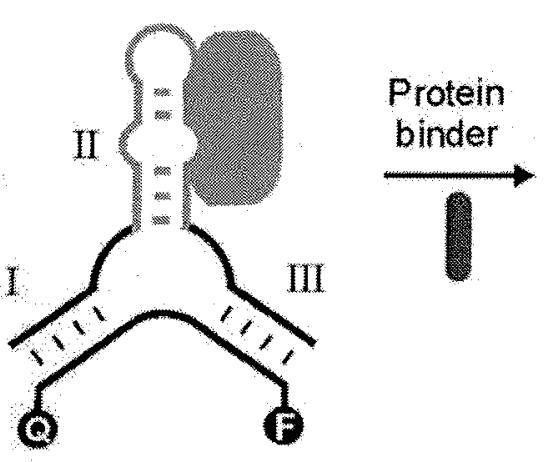
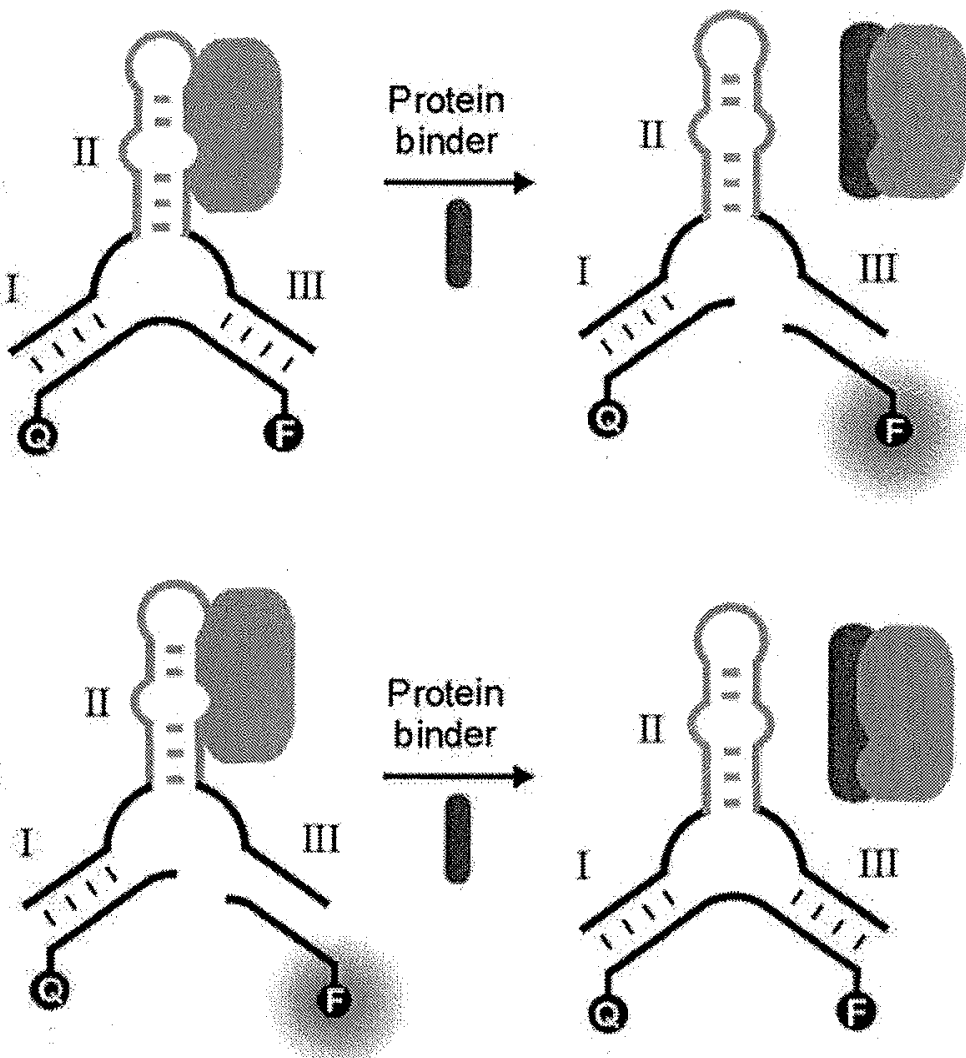

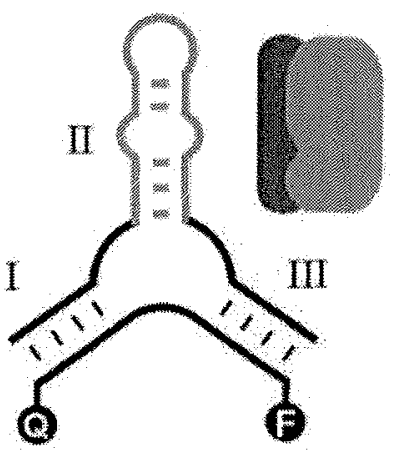

Fig. 2. General outline for the detection of protein interactions using reporter ribozymes. (A) Starting from catalytically active ribozymes. The ribozyme is switched off by the target protein via interaction with a protein-binding RNA domain (light grey). In the protein-bound state (middle), the complex can be used as sensor for interaction partners of the protein. In the presence of a protein binder (dark grey) that competes with the ribozyme binding of the protein, the ribozyme is liberated and catalytic activity is switched on. (B) Starting from inactive ribozymes: cleavage activity is induced by the target protein, addition of the interaction partner of the protein leads to inactivation of the ribozyme. When aiming at the detection of interaction partners of the target protein, the first approach is preferred since it generates a positive readout if an interaction takes place. If solely the detection of the presence or concentration of the target protein alone is desired, the second setup should be used since a signal is generated upon presence of the target protein.

thereby blocking substrate binding and hence cleavage activity (see Fig. 3A). Upon addition of HIV-1 Rev, the attached aptamer sequence changes its conformation driven by protein binding. Hence, the protein interaction allows the substrate to access the $5^{\prime}$-end of the ribozyme, thereby facilitating its cleavage. In a second design, the naturally occurring HIV-1 Rev-binding RNA sequence (Rev-binding element) was attached to the ribozyme in place of helix II (5). Upon addition of Rev protein, this ribozyme was switched off, displaying catalytic 

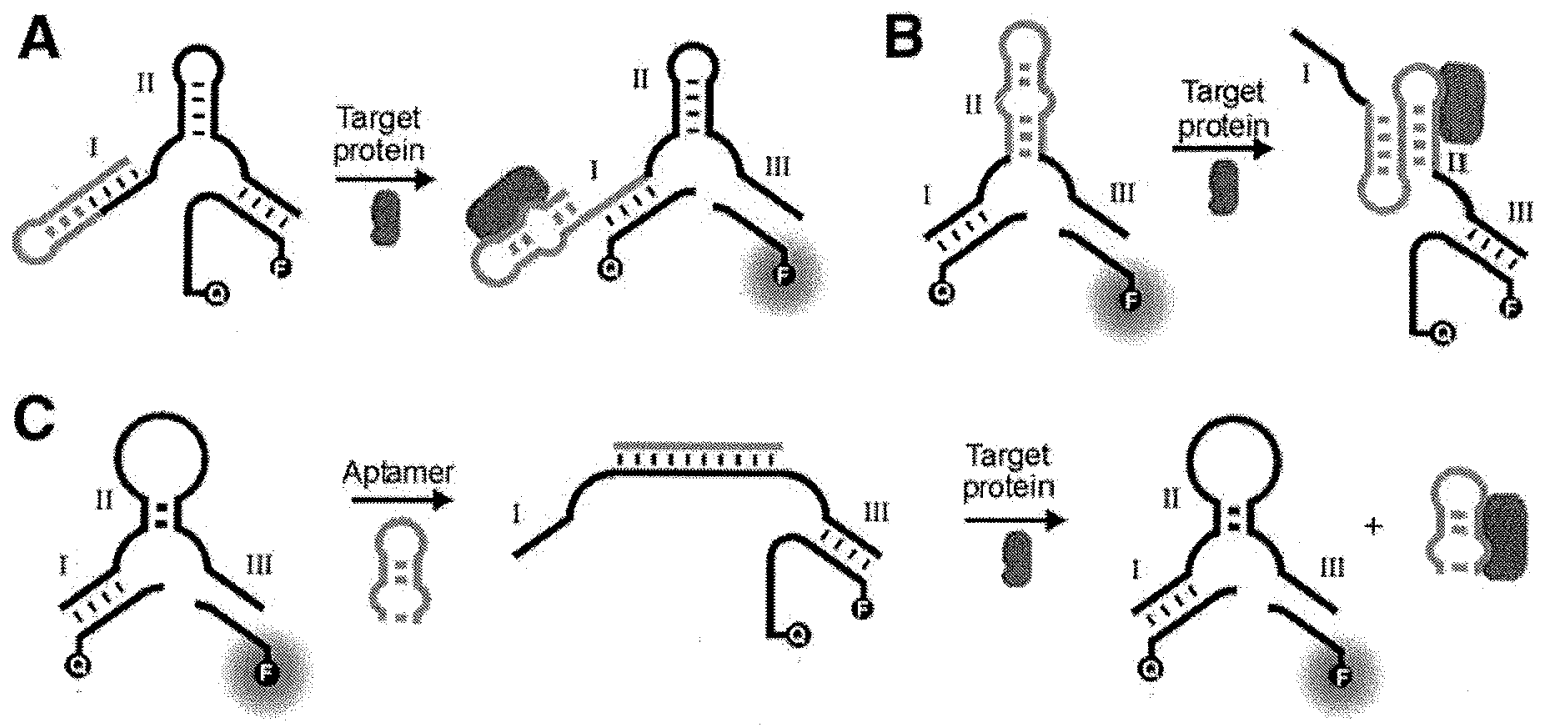

Fig. 3. Examples of protein-responsive ribozymes. Aptamer sequences are indicated in light grey. (A) Reporter ribozymes constructed by fusion of an aptamer to a ribozyme. The fused aptamer sequence prevents binding of the ribozyme to its substrate. Addition of the target protein results in folding of the aptamer and liberation of the substratebinding site enabling cleavage activity. (B) In a second approach, aptamers undergoing structural reorganization upon binding of the target protein are fused to the hammerhead ribozyme to build stem II. For example, protein binding induces a pseudo-knotted structure of the aptamer, thereby disrupting the formation of the catalytic core (right panel). (C) This approach differs significantly from the others since a sequence complementary to the aptamer, instead of the aptamer itself, is introduced into the ribozyme (dark grey). Addition of the free aptamer (light grey) results in its hybridization to stem II of the ribozyme, resulting in disruption of the catalytically active ribozyme fold. If the target protein is added, the aptamer is decoyed from the ribozyme when complexing its target, thereby releasing the catalytically active ribozyme.

behavior opposite to that of the first Rev-responsive ribozyme. These ribozymes were subsequently used to screen a structurally diverse library of antibiotics for Rev-binding activity. If the Rev protein is bound by the small molecule, the ribozyme gets displaced from the complex and the catalytic activity changes. The experiment demonstrated the suitability of aptazymes for high-throughput assays and identified the first small molecules that bind directly to HIV-1 Rev. One of the identified antibiotics was subsequently shown to repress HIV-1 replication in cell culture (5).

\subsubsection{Detecting Interactions of HIV-1 Reverse Transcriptase}

A different strategy for constructing protein-responsive ribozymes was exploited when we generated an aptazyme controlled by reverse transcriptase 
(RT) of HIV-1. The aptamer for HIV-1 RT folds into a pseudoknot structure when complexed with the protein (see Refs. [14-16]). A competing, non-binding fold of the aptamer sequence is represented by a hairpin-looped structure. As shown in Fig. 3B, the aptamer was inserted into stem II of the HHR, resulting in a fusion construct FK-1 with competing folds of the ribozyme and the aptamer pseudo-knotted structure (17). The simultaneous folding of both domains is impossible in this design, since pseudoknot formation results in disruption of the catalytic core of the ribozyme. In the absence of HIV-1 RT, the ribozyme folds and displays cleavage activity, with the inserted aptamer sequence folded as a hairpin loop in stem II (see Fig. 3B, left). Upon binding of HIV-1 RT, the pseudo-knotted fold of the aptamer is induced, disrupting stem II, and thus inhibiting catalytic activity of ribozyme FK-1. The ribozyme senses the presence of the protein at concentrations as low as $1 \mathrm{n} M$ with a half-maximum inhibition of cleavage activity occurring at $10 \mathrm{n} M \mathrm{HIV}-1 \mathrm{RT}$. In addition to high sensitivity, the ribozyme displays high specificity since the reporter is unaffected by the presence of the homologue RT of HIV-2.

By generating variants of the reporter ribozyme for HIV-1 RT, we were able to demonstrate that rational design can be used to fine-tune nucleic acid function. Deletion of a single GC base pair in stem II yielded a construct with a destabilized stem II, which showed no cleavage activity in the absence of protein. In contrast, insertion of an additional GC base pair stabilizes stem II. This mutant is catalytically active, with the cleavage activity being unaffected even by the presence of HIV-1 RT. Owing to the increased stability of stem II, the protein is no longer able to induce folding of the pseudoknot. The generation of such reporter variants demonstrates that ribozyme properties can be designed by rational means (17).

Interaction partners of HIV-1 RT were detected using the aptazyme FK-1. A complex of double-stranded DNA, which competes with the aptazyme for binding of HIV-1 RT, was detected in a concentration-dependent manner. In addition, drugs binding to the primer-template domain of the polymerase were detected as well. Interestingly, a RT inhibitor that also binds to the protein, but at a different site from the primer-template site was not detected, suggesting a domain-specific mode of detection (17).

\subsubsection{Detecting Interactions of Human $\alpha$-Thrombin}

A different approach was taken when we generated RNA reporters capable of detecting human $\alpha$-thrombin and interaction partners (5). The strategies for rational design discussed so far are best suited for generating reporters composed of natural RNA. Modified RNA or DNA aptamers are difficult to employ in such strategies since they cannot be introduced by standard in vitro transcription. In Fig. 3C, a novel strategy is introduced, allowing the use of aptamers composed of nucleic acids other than natural RNA. A ribozyme detecting human $\alpha$-thrombin 
was generated by incorporating the RNA sequence complementary to the anti-thrombin DNA aptamer in stem II of the HHR (see Fig. 3C). The ribozyme itself is catalytically active. Upon addition of the DNA aptamer, hybridization to stem-loop II of the ribozyme prevents the formation of the catalytic core, thus switching the ribozyme off. Cleavage activity is restored by addition of $\alpha$-thrombin, which complexes the DNA aptamer, thus releasing the active conformation of the ribozyme (see Fig. 3C, right). This protein-dependent release of the ribozyme turned out to be highly specific for human $\alpha$-thrombin. Related clotting factors as well as less closely related proteins were unable to restore cleavage activity (5).

The thrombin-dependent ribozyme was used to detect the interaction between $\alpha$-thrombin and a naturally occurring inhibitor peptide (hirudin) isolated from the leech Hirudo medicinales. Even small alterations of the analyte could be detected. For example, modification of hirudin by a single sulfate group was sensed (5). Moreover, the interaction between thrombin and a second interaction partner (anti-thrombin III) was not sensed. Since anti-thrombin III binds to thrombin at a different site than both hirudin and the DNA aptamer, interference with the aptamer binding did not occur. This result again shows that detection is likely based on competition of binding, enabling the detection of interactions in a domain-specific fashion.

\section{Materials}

\subsection{Reagents and Solutions}

Since the methodology utilizes RNA as reporters, extreme care should be dedicated to work with ribonuclease-free solutions and reagents. Buffers and solutions should exceptionally be prepared with DEPC-treated water. Additional measures should be taken in order to prevent contamination, see also (18).

HHR catalysis is effective under a wide range of conditions and will tolerate variations in buffer composition, temperature, and salt concentrations. The buffer can therefore be chosen to meet the specific requirements of the target protein. The buffer of choice should not contain divalent metal ions such as $\mathrm{Mg}^{2+}$ or $\mathrm{Ca}^{2+}$, since these are added separately in order to initiate ribozyme cleavage.

\subsection{Synthesis of Aptazymes and Substrate}

Aptazymes are best prepared by in vitro transcription starting from a dsDNA template. Large quantities of the template can be obtained by PCR-amplification of the corresponding DNA sequence, containing a T7 promoter for in vitro transcription. The ribozyme itself is then purified from the subsequent in vitro transcription reaction by standard denaturing polyacrylamide gel electrophoresis (PAGE). For details of methodology, see Chapter 12. 
Ribozyme sequences shorter that 35 nucleotides are best prepared using solid phase phosphoramidite chemistry since transcription yields tend to be low (19). This technique also allows the synthesis of the RNA substrate modified with the two reporter dyes, which subsequently enable fluorescence readout of the cleavage reaction (20). Many companies offer customized synthesis of modified or natural RNA (see http://www.glenres.com/ExtraPages/oligohouses.html).

\section{Methods}

\subsection{Aptamer Selection}

The first step of constructing a protein-responsive ribozyme is the selection of a nucleic acid sequence that binds to the target protein. This sequence might have a natural origin, as discussed above in the case of the ribozyme inhibited by the presence of HIV Rev (5). In this example, a sequence that specifically binds to an arginine-rich domain of the Rev protein was adapted from the RNA genome of HIV in order to render the ribozyme Rev-dependent. If a naturally occurring nucleic acid is not available for the protein of interest, then the systematic evolution of ligands by exponential enrichment procedure can be used to generate protein-binding nucleic acid sequences (21-23).

\subsection{Design of Potential Aptazymes}

If an RNA sequence that binds to the protein of interest has been identified, fusions of this sequence and the HHR can be designed based on the principles discussed above. Fusion sequences have to be examined for the desired secondary structures using $m$ fold or similar folding algorithms prior to aptazyme synthesis (24). Evaluating the secondary structure of potential aptazymes by such algorithms represents a powerful tool for predicting the catalytic behavior of a designed sequence. Although these algorithms do not take into account binding energies resulting from protein interactions or possible tertiary structure motifs, they often enable reliable prediction of the cleavage activity of the ribozyme in the absence of the target protein. For example, to generate a ribozyme that possesses catalytic activity, the secondary structure of the catalytic core, shown in Fig. 1A, should be among the lowest folding energy predictions generated by mfold or other algorithms (see Note 1). If promising candidates are found, synthesis of substrate and ribozyme RNA should be carried out as described in the Materials section.

\subsection{Characterization of Aptazyme Sequences}

In order to test whether the constructed ribozyme sequences respond to the presence of the target protein with a change in catalytic activity, cleavage reactions are carried out in the presence and absence of the target protein (see Note 2). 
If the examined ribozymes show pronounced (10-fold or more) differences in catalytic activity in the presence and absence of the target protein, they are suited for screening for interactions of the target protein. The identification of a ribozyme sequence that is unaffected by the presence of the target protein is not without its uses. Such non-responsive ribozymes can be used in control reactions during the final screening experiment, as described in Section 3.4.

Reactions for the characterization of ribozymes can be carried out in multi-well plate fluorescence readers, using 96- or 384-well plates, in volumes as low as $10 \mu \mathrm{L}(8)$. Nevertheless, for easy handling, a total reaction volume of $50 \mu \mathrm{L}$ is appropriate. The ribozyme reaction is initiated by adding $\mathrm{MgCl}_{2}$ (see Note 3 ) to a final concentration of $10 \mathrm{~m} M$ (see Note 4). During the course of the reaction, fluorescence is measured as a function of time. Especially in cases where the ribozyme reaction appears to be relatively slow ( $\left.\kappa_{\mathrm{obs}}<<1 \mathrm{~min}\right)$, data acquisition should be carried out at reasonable intervals, e.g., every 2-5 min since excessive excitation of the fluorescent markers results in bleaching of the dyes (see Note 5).

Typical starting reaction conditions are as follows:

Final volume: $50 \mu \mathrm{L}$

\begin{tabular}{ll} 
Combine & Final concentration \\
\hline Buffer & As required \\
Ribozyme & $50 \mathrm{n} M$ \\
Substrate & $500 \mathrm{n} M$ \\
Target protein & $50 \mathrm{n} M-5 \mu M$ \\
$\mathrm{MgCl}_{2}$ & $10 \mathrm{~m} M$ (reaction start) \\
\hline
\end{tabular}

Prior to starting the ribozyme reactions with $\mathrm{MgCl}_{2}$, the ribozyme, RNA substrate, target protein, and analyte should be pre-incubated for an appropriate time to allow formation of the desired complexes (see Note 6).

\subsection{Screening and Evaluation}

If a protein-responsive ribozyme is identified, analytes can be screened for interactions with the target protein. The individual reactions should be performed in a multi-well plate format, including duplicate standard reactions of the ribozyme alone, as well as ribozyme with target protein on the same well plate. Ribozyme-only reactions (lacking protein) are used to normalize the data obtained from reactions containing potential interaction partners.

Two independent reactions should be carried out for each analyte tested: one containing the reporter ribozyme and the target protein, and one containing target protein and a ribozyme that is not regulated by the protein (see Section 3.3.). The latter reaction serves as a control to identify analytes that influence ribozyme activity by interacting directly with the ribozyme rather than with the target protein. When this control is factored in, all influences affecting solely 
the ribozyme activity can be excluded. The target-insensitive control ribozyme should display cleavage activity independent of the target protein. For this purpose, variants of the aptazyme-containing scrambled sequences or mutations in the aptamer part of the molecule generally work well since such changes usually prevent interactions with the target protein. If such a mutant is not easily found, unmodified ribozymes such as shown in Fig. 1A may serve as control $(7,25)$.

Strong ribonuclease activity in the analyte preparations represents a challenge and should be avoided. Ribonuclease activity results in digestion of the RNA substrate, which could be misinterpreted as cleavage activity originating from the ribozyme. Nevertheless, weak-to-moderate nuclease activity is tolerable if a control reaction for each analyte, containing only the RNA substrate, is included. By doing so, correction of the ribozyme cleavage data is possible by subtracting the "substrate only" control.

The following equation can be used to evaluate the raw cleavage data:

$$
A_{\text {rel }}=\frac{A_{\text {analyte }}^{\text {reporter }} / A_{\text {standard }}^{\text {reporter }}}{A_{\text {analyte }}^{\text {control }} / A_{\text {standard }}^{\text {control }}}
$$

With

$A_{\text {analyte }}^{\text {report }}$ : Activity of reporter ribozyme in presence of target protein and analyte;

$A_{\text {standard }}^{\text {repoter }}$ Activity of reporter ribozyme in presence of target protein only;

$A_{\text {analyte }}^{\text {control }}$ : Activity of control ribozyme in presence of target protein and analyte;

$A_{\text {standard }}^{\text {control }}$ : Activity of control ribozyme in presence of target protein only.

The value that is described by this equation represents a relative activity, corrected for nonspecific influences affecting the ribozyme directly, and thus revealing true interaction partners of the target protein. The interaction partners identified by the aptazyme-based screening protocol should be validated using alternate methods for characterizing biomolecular interactions.

\section{Notes}

1. The aptazyme is designed to function as an in trans-cleaving ribozyme. The substrate sequence should be included when calculating the secondary structure of a potential ribozyme. Since folding of a single sequence is achieved more easily, we connect the substrate and ribozyme strand via a short loop (e.g., a GNRA tetraloop, with N: any nucleotide; R: G or A), as shown in Fig. 1A when calculating the lowest energy fold. 
2. The optimal ratio of ribozyme to substrate will vary with the catalytic fitness of the ribozyme, but ribozyme concentrations ranging from 5 to $100 \mathrm{nM}$ with substrate concentrations between 200 and $1000 \mathrm{n} M$ are a good starting point. An excess of substrate enables signal amplification, since the in trans versions of the ribozymes used here are able to perform multiple substrate turnovers.

3. The reactions can be performed in a highly parallel fashion by simultaneously adding $\mathrm{MgCl}_{2}$ to all reaction wells. The reaction can be started manually by adding $\mathrm{MgCl}_{2}$ using a multi-channel pipette since the ribozyme reactions are slow when compared with most protein kinetics. The microtiter plate should then be assayed immediately using the fluorescence reader. Nevertheless, we usually use an automated dispensing device of the fluorescent reader to start the ribozyme reactions.

4. The concentration of $\mathrm{Mg}^{2+}$ required for optimum cleavage activity could be influenced by the modification of the ribozyme by fusing the aptamer sequence. Ten millimolar of $\mathrm{MgCl}_{2}$ is usually appropriate for unmodified HHRs and works well for most modified ribozymes.

5. Alternatively, ribozyme catalysis can be monitored by assessing cleavage of radioactively labeled RNA substrates. This might be helpful if the analytes are fluorescent and interfere with the fluorescent readout. Nevertheless, the high-throughput character of the method is lost when using radioactively labeled substrates.

Aliquots are removed at certain time points during the reaction and are quenched in formamide loading buffer. Cleavage products are separated from the uncleaved substrate by $12 \%$ PAGE, and subsequently analyzed by autoradiography. The fraction of cleaved substrate is divided by the sum of cleaved and intact substrate, and plotted versus the time, yielding a time course of the cleavage reaction.

6. The order of addition of the components usually has little influence on the resulting ribozyme activity, although comparison of different orders of addition, as well as a range of incubation times can be carried out for optimizing the reaction.

\section{Acknowledgments}

This work was supported by the Deutsche Forschungsgemeinschaft and the Volkswagen-Stiftung (to M.F.).

\section{References}

1. Breaker, R. R. (2002) Engineered allosteric ribozymes as biosensor components. Curr. Opin. Biotechnol. 13, 31-39.

2. Seetharaman, S., Zivarts, M., Sudarsan, N., and Breaker, R. R. (2001) Immobilized RNA switches for the analysis of complex chemical and biological mixtures. Nat. Biotechnol. 19, 336-341.

3. Jenne, A., Gmelin, W., Raffler, N., and Famulok, M. (1999) Real-Time Characterization of Ribozymes by Fluorecence Resonance Energy Transfer (FRET). Angew. Chem. Int. Ed. 38, 1300-1303.

4. Jenne, A., Hartig, J. S., Piganeau, N., et al. (2001) Rapid identification and characterization of hammerhead-ribozyme inhibitors using fluorescence-based technology. Nat. Biotechnol. 19, 56-61. 
5. Hartig, J. S., Najafi-Shoushtari, S. H., Grune, I., Yan, A., Ellington, A. D., and Famulok, M. (2002) Protein-dependent ribozymes report molecular interactions in real time. Nature Biotechnology 20, 717-722.

6. Hertel, K. J., Stage-Zimmermann, T. K., Ammons, G., and Uhlenbeck, O. C. (1998) Thermodynamic dissection of the substrate-ribozyme interaction in the hammerhead ribozyme. Biochemistry 37, 16,983-16,988.

7. Stage-Zimmermann, T. K. and Uhlenbeck, O. C. (1998) Hammerhead ribozyme kinetics. Rna 4, 875-889.

8. Hartig, J. S., Grune, I., Najafi-Shoushtari, S. H., and Famulok, M. (2004) Sequence-specific detection of MicroRNAs by signal-amplifying ribozymes. J. Am. Chem. Soc. 126, 722-723.

9. Koizumi, M., Soukup, G. A., Kerr, J. N., and Breaker, R. R. (1999) Allosteric selection of ribozymes that respond to the second messengers cGMP and cAMP. Nat. Struct. Biol. 6, 1062-1071.

10. Soukup, G. A. and Breaker, R. R. (1999) Engineering precision RNA molecular switches. Proc. Natl Acad. Sci. USA 96, 3584-3589.

11. Piganeau, N., Thuillier, V., and Famulok, M. (2001) In vitro selection of allosteric ribozymes: theory and experimental validation. J. Mol. Biol. 312, 1177-1190.

12. Piganeau, N., Jenne, A., Thuiller, V., and Famulok, M. (2001) An allosteric ribozyme regulated by doxycycline. Angew. Chem. Int. Ed. 39, 4369-4373.

13. Kertsburg, A. and Soukup, G. A. (2002) A versatile communication module for controlling RNA folding and catalysis. Nucleic Acids Res. 30, 4599-4606.

14. Tuerk, C., MacDougal, S., and Gold, L. (1992) RNA pseudoknots that inhibit human immunodeficiency virus type 1 reverse transcriptase. Proc. Natl Acad. Sci. USA 89, 6988-6992.

15. Jaeger, J., Restle, T., and Steitz, T. A. (1998) The structure of HIV-1 reverse transcriptase complexed with an RNA pseudoknot inhibitor. Embo. J. 17, 4535-4542.

16. Kensch, O., Connolly, B. A., Steinhoff, H. J., McGregor, A., Goody, R. S., and Restle, T. (2000) HIV-1 reverse transcriptase-pseudoknot RNA aptamer interaction has a binding affinity in the low picomolar range coupled with high specificity. J. Biol. Chem. 275, 18,271-18,278.

17. Hartig, J. S. and Famulok, M. (2002) Reporter ribozymes for real-time analysis of domain-specific interactions in biomolecules: HIV-1 reverse transcriptase and the primer-template complex. Angew. Chem. Int. Ed. 41, 4263-4266.

18. Sambrook, J. and Russell, D. W. (2001) In Molecular Cloning, Vol. 1, p. 7.82, Cold Spring Harbor Laboratory Press, Cold Spring Harbor, New York.

19. Beaucage, S. L. (2002) Synthesis of Unmodified Oligonucleotides, In Current Protocols in Nucleic Acid Chemistry (Beaucage, S. L., Bergstrom, D. E., Glick, G. D., Jones, R. A., eds.), John Wiley \& Sons, Chapter 3.

20. Beaucage, S. L. (2003) Synthesis of Modified Oligonucleotides and Conjugates. In Current Protocols in Nucleic Acid Chemistry (Beaucage, S. L., Bergstrom, D. E., Glick., G. D., Jones, R. A, eds.), John Wiley \& Sons, Chapter 4.

21. Sun, S. (2000) Technology evaluation: SELEX, Gilead Sciences Inc. Curr. Opin. Mol. Ther. 2, 100-105. 
22. Joyce, G. F. (1994) In vitro evolution of nucleic acids. Curr. Opin. Struct. Biol. 4, 331-336.

23. Klug, S. J. and Famulok, M. (1994) All you wanted to know about SELEX. Mol. Biol. Rep. 20, 97-107.

24. Zuker, M. (2003) Mfold web server for nucleic acid folding and hybridization prediction. Nucleic Acids Res. 31, 3406-3415.

25. Hertel, K. J., Herschlag, D., and Uhlenbeck, O. C. (1994) A kinetic and thermodynamic framework for the hammerhead ribozyme reaction. Biochemistry 33, 3374-3385. 